\title{
Evaluation of sucrose as carbon source in mixotrophic culture of Arthrospira platensis Gomont 1892
}

\author{
Zülfiye Velioğlu Tosuner ${ }^{1}$ (D), Raziye Öztürk Ürek ${ }^{2}$
}

Cite this article as:

Velioğlu Tosuner, Z., Öztürk Ürek, R. (2020). Evaluation of sucrose as carbon source in mixotrophic culture of Arthrospira platensis Gomont 1892. Aquatic Research, 3(1), 1-12. https://doi.org/10.3153/AR20001

${ }^{1}$ Dokuz Eylül University, Graduate School of Natural and Applied Sciences, Department of Biotechnology, 35160 Buca, Izmir, Turkey

${ }^{2}$ Dokuz Eylül University, Faculty of Science, Department of Chemistry, 35160 Buca, Izmir, Turkey

ORCID IDs of the author(s): Z.V.T. 0000-0001-9181-6619 R.Ö.Ü. 0000-0002-7147-6853

Submitted: 07.08.2019

Revision requested: 03.09.2019

Last revision received: 04.10 .2019

Accepted: 06.10.2019

Published online: 25.10 .2019

Correspondence:

Zülfiye VELIOĞLU TOSUNER

E-mail: zulfivevelioglu@gmail.com

\begin{abstract}
Cyanobacteria are photosynthetic microorganisms that use $\mathrm{CO}_{2}$ as carbon source and sunlight as energy source. Although phototrophic cultivation is widely used in cyanobacterium production, heterotrophic and mixotrophic cultivations attract attention among researchers. In this study the effect of different concentrations ( 0 [control] - $0.25-2.5-10-50 \mathrm{mM})$ of sucrose on the growth of Arthrospira platensis under mixotrophic cultivation was investigated. The purpose of this study was to investigate whether $A$. platensis biomass production could be performed regardless of high light intensity. Biomass, chlorophyll, lipid and carbohydrate contents were determined by spectrophotometrically. Also the physicochemical properties of the produced cyanobacterium were investigated by FTIR, TGA and DSC. The highest biomass productivity was detected as 1.33 $\mathrm{g} / \mathrm{L} /$ day in the medium containing $2.5 \mathrm{mM}$ sucrose and the specific growth rate increased 1.32 fold as compared to phototrophic culture. Additionally, the highest lipid content ( $3.68 \pm 0.17 \mathrm{mg} / \mathrm{g}$ cell) was determined in the same medium. This suggests that $A$. platensis has adapted to the medium that contains low sucrose concentrations. Also, this study showed that sucrose containing medium supports lipid production.
\end{abstract}

Keywords: Sucrose, Mixotrophic culture, Phototrophic culture, Arthrospira platensis, Lipid production

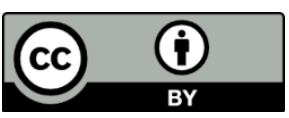

(C)Copyright 2020 by ScientificWebJournals

Available online at 


\section{Introduction}

Cyanobacteria are photosynthetic microorganisms that use $\mathrm{CO}_{2}$ as a carbon source and sunlight as energy (Katiyar et al., 2017; Patel et al., 2017). In laboratory scale this natural production type is called phototrophic culture. Although phototrophic cultivation does not need organic carbon source, this culture type makes slow cell growth, low biomass, and higher harvesting cost (Gim et al., 2016; Ozturk Urek \& Kerimoglu, 2019). While phototrophic cultivation is widely used in the laboratory, pilot and industrial scale, cyanobacterium cultivation is also performed in heterotrophic cultivation, which contains an external carbon source (Joannesa et al., 2016). In heterotrophic cultivation, cyanobacterium cells are grown in the presence of external carbon source (acetate, glucose, sucrose etc.) but no light (Meireles et al., 2017). The medium in which both $\mathrm{CO}_{2}$ and an external organic carbon source are present as carbon sources are mixotrophic cultivations. Some microalgae, such as Chlorella regularis, Nannochloropsis sp., Synechococcus sp., Anabaena sp., Arthrospira platensis, can grow better under mixotrophic condition, which may combine the advantages of phototrophic and heterotrophic cultures (Zhan et al., 2017). The advantages of mixotrophic cultivation are higher growth rate, higher biomass and lipid accumulation, sustain of pigmentation and phytochemicals production, decreased production of $\mathrm{CO}_{2}$ while there are some problems such as higher cost because of organic carbon source, contamination risk, and reduced energy conversion efficiency (Van Wagenen et al., 2015; Wang et al., 2017; Zhan et al., 2017). When compared with heterotrophic culture, the biomass production in mixotrophic cultivation is not only dependent on the carbon source type and amount. Similarly, in mixotrophic cultivation, there is no need for light intensity as high as in phototrophic cultivation, and light dependence is lower (Abreu et al., 2012). As in mixotrophic growth cyanobacteria showed different metabolic activity from phototrophic culture. Photosynthesis and aerobic respiration are stimulated simultaneously in mixotrophic cultures. Mixotrophic growth offers increasing microbial cell concentration in addition to protein, carbohydrate and lipid productivity. Therefore, mixotrophic cultivation is more economical and easier to control than the other two cultivation types.

In a study growth, lipid and biomass productivity of Chlorella vulgaris and Leptolyngbya sp. in heterotrophic and mixotrophic regimes were investigated (Silaban et al., 2014). Dextrose and sodium acetate were used as external carbon source and the highest biomass productivity $\left(156 \mathrm{~g} / \mathrm{m}^{3} \mathrm{~d}\right)$ and neutral lipid productivity $\left(24.07 \mathrm{~g} / \mathrm{m}^{3} \mathrm{~d}\right)$ was detected with $2.1 \mathrm{~g} / \mathrm{L}$ sodium acetate in mixotrophic culture. In a study of Ceron Garcia et al., (2006) Phaeodaciylum tricornutum was grown in mixotrophic culture which contains fructose, glucose, mannose, lactose or glycerol as external carbon source. Glycerol $(0.1 \mathrm{M})$ was detected as the best substrate that increased final biomass level by 7 fold relative to control cultures. $A r-$ throspira platensis cyanobacterium and Chlorella homosphaera microalgae were cultivated with glucose in mixotrophic conditions and resulting in biomass increases of up to 3.45 and 2.79 fold, respectively (Margarites et al., 2017).

Since the cyanobacteria Arthrospira sp. has important nutritional properties with high protein, essential amino acid and vitamin content, it is an important fish diet alternative (Rosas et al., 2018; Sivakumar et al., 2018). Arthrospira sp. can utilize organic carbon substrates in heterotrophic and mixotrophic conditions (Marquez et al., 1993). The blue-green algae $A$. platensis grows mainly on inorganic carbon source and much work has not been carried out on the utilization of organic carbon sources. Some of monosaccharides and disaccharides such as glucose, fructose, sucrose and lactose have been used for mixotrophic cultivation of cyanobacterium and different transport and assimilation mechanisms may be effective for each sugar (Chojnacka \& Marquez-Rocha, 2004).

A. platensis is suitable as a biotreatment material for fish production effluents which shows adaptation in mixotrophic cultures. In a study, A. platensis was inoculated to the fish culture effluent in order to remove the dissolved nutrients (Nogueira et al., 2018). The concentration of ammonia, nitrite, nitrate and phosphate was detected lower by more than $94.8 \%$, and maximum $A$. platensis productivity was determined as $0.03 \mathrm{~g} / \mathrm{L}$. day.

In the study of Chojnacka and Noworyta (2004), the influence of growth parameters on specific growth rate of Arthrospira sp. in photoautotrophic, heterotrophic and mixotrophic batch modes were investigated and the highest specific growth rate $\left(0.055 \mathrm{~h}^{-1}\right)$ was reached in mixotrophic culture with $2.5 \mathrm{~g} / \mathrm{L}$ glucose.

Some studies have thus focused on finding cheaper organic carbon sources to decrease production cost (Bhatnagar et al., 2011; Lin \& Wu, 2015). Sucrose present in the waste of the sugar production process is an important alternative carbon source (Abreu et al., 2012; Wang et al., 2016). The use of sucrose-containing waste as a carbon source will evaluate of a waste material and provide low cost production (Mitra et al., 2012). Therefore, it is important to investigate the growth and production aspects of cyanobacterium in sucrose containing medium. 
In this study $A$. platensis was cultivated under mixotrophic cultivation with different concentrations of sucrose as a carbon source. Effects of carbon source's concentration on production of biomass, chlorophyll, and total lipid were investigated. Also specific growth rates were calculated. The aim of this study was to investigate the effect of sucrose concentration on growth and lipid production of $A$. platensis. The lipid production of $A$. platensis in sucrose-containing growth medium was investigated for the first time in this study. Also the characterization of produced cyanobacterium cell with TGA and FTIR is a novel approach.

\section{Material and Methods}

\section{Cyanobacteria and Culture Media}

The cyanobacteria Arthrospira platensis (Gomont) 1892 was provided from Cukurova University, Faculty of Aquaculture, Adana-Turkey. For the maintenance of cyanobacteria under phototrophic culture, it has been growth in Zarrouk's medium (Zarrouk, 1966). Batch cultivation was carried out in $750 \mathrm{~mL}$ medium at 2500 lux $\left(33.75 \mu \mathrm{mol}\right.$ photon $\left.\mathrm{m}^{-2} \mathrm{~s}^{-2}\right)$ light intensity (by white fluorescent lamps) with continuous illumination, $\mathrm{pH} 9.0$ and $30^{\circ} \mathrm{C}$ and the cultures were mixed and aerated using filtered air continuously.

\section{Mixotrophic Cultivation}

Mixotrophic culture was carried out in Zarrouk's medium, which contained different concentration of sucrose ( 0 [control] $-0.25-2.5-10-50 \mathrm{mM}$ ) as carbon source. Culture was inoculated to an initial optical density (OD) of 0.2 at 600 $\mathrm{nm}$ (Vonshak et al., 1982). OD is a parameter used to determine biomass production. When working with filamentous microorganisms, make sure that the culture medium is well mixed before reading the OD. In this present study, well mixed A. platensis culture was transferred to spectrophotometer cuvette and the cuvette was turned upside down for three timed and then OD was read.

Batch cultivation was carried out in $250 \mathrm{~mL}$ Erlenmeyer with $100 \mathrm{~mL}$ working volume at $1500 \mathrm{lux}(20.25 \mu \mathrm{mol}$ photon $\mathrm{m}^{-2} \mathrm{~s}^{-2}$ ) light intensity (by white fluorescent lamps) with continuous illumination, $100 \mathrm{rpm}$ shaking rate (Thermoshake Incubator, Gerhardt, Germany), $\mathrm{pH} 9.0$ and $30^{\circ} \mathrm{C}$. OD, $\mathrm{pH}$, chlorophyll and total lipid content were detected during incubation period. Zarrouk's medium without any external carbon sources was used as control condition.

Specific growth rate $(\mu)$ and biomass productivity $(\mathrm{P})$ were calculated according to Eq. 1 and 2 based on OD values (Kong et al., 2013) (X: amount of microorganism, t: time as day).
$\mu=\ln \frac{\mathrm{X} 1-\mathrm{X} 0}{\mathrm{t} 1-\mathrm{t} 0}$
Eq. 1
$\mathrm{P}=\frac{\mathrm{X} 1-\mathrm{X} 0}{\mathrm{t} 1-\mathrm{t} 0}$
Eq. 2

\section{Determination of Chlorophyll Content}

Chlorophyll a and b contents were measured as described by Lichtenthaler and Wellburn (1983). $5 \mathrm{~mL}$ of algal suspension was centrifuged at $5000 \mathrm{rpm}$ for $15 \mathrm{~min}$. Pellet was weighted and homogenized in $5 \mathrm{~mL}$ absolute ethanol by $8000 \mathrm{rpm}$ for $1 \mathrm{~min}$ and $9500 \mathrm{rpm}$ for 1 min with 30 seconds intervals with laboratory homogenizer (Ultra Turrax, IKA, Germany). After centrifugation absorbance of the obtained supernatant was measured at 470, 664.2 and $648.6 \mathrm{~nm}$. Chlorophyll $\mathrm{a}$ and $\mathrm{b}$ contents were calculated according to Eq. 3 and 4 (Lichtenthaler \& Wellburn, 1983).

$$
\begin{array}{ll}
\mathrm{Chl} \mathrm{a}=13.36 \times \mathrm{Abs}_{664.2}-5.19 \times \mathrm{Abs}_{648.6} & \text { Eq. } 3 \\
\mathrm{Chl} \mathrm{b}=27.43 \times \mathrm{Abs}_{648.6}-8.12 \times \mathrm{Abs}_{664.2} & \text { Eq. } 4
\end{array}
$$

\section{Determination of Total Lipid Content}

Total lipid content of cyanobacterium was determined by Mishra et al., (2014) method. To prepare reagent $0.6 \mathrm{~g}$ vanillin was dissolved in $10 \mathrm{~mL}$ ethanol and mixed $90 \mathrm{~mL}$ distilled water and $400 \mathrm{~mL}$ concentrated phosphoric acid. $2 \mathrm{~mL}$ concentrated sulfuric acid was added to $100 \mu \mathrm{L}$ cyanobacteria sample and was heated for $10 \mathrm{~min}$ at $100^{\circ} \mathrm{C}$, and was cooled for $5 \mathrm{~min}$ in ice bath. $5 \mathrm{~mL}$ of freshly prepared phospho-vanillin reagent was then added and the sample was incubated for $15 \mathrm{~min}$ at $37^{\circ} \mathrm{C}$ incubator shaker at $150 \mathrm{rpm}$. The absorbance was measured at $530 \mathrm{~nm}$ against a reference sample.

\section{Determination of Total Carbohydrate Content}

Total carbohydrate content of production medium was determined by phenol-sulphuric acid method (Dubois et al., 1956). To determine total carbohydrate content, $1 \mathrm{~mL}$ cell free supernatant ( $1 \mathrm{~mL}$ distilled water for reference) was mixed with

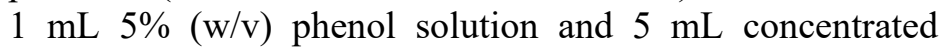
$\mathrm{H}_{2} \mathrm{SO}_{4}$. After well mixing the samples were incubated for 20 $\mathrm{min}$ at room temperature the absorbance was measured at 470 $\mathrm{nm}$ against a reference sample. Glucose was used as standard in the range of $0-250 \mu \mathrm{g} / \mathrm{mL}$.

\section{TGA and FTIR Analysis}

TGA and DSC analyses of produced cyanobacterium in phototrophic and mixotrophic cultures were carried out with Perkin Elmer- Diamond TG/DTA (Massachusetts, USA). About 3-5 mg of dry produced cyanobacterium cell sample was loaded on a platinum pan and its energy level was scanned in 
the ranges of $30-500^{\circ} \mathrm{C}$ under a nitrogen atmosphere with a temperature gradient of $10^{\circ} \mathrm{C} / \mathrm{min}$.

To analyze the organic structure of produced A. platensis cell, the FT-IR spectra were recorded on the Perkin Elmer Spectrum BX (Massachusetts, USA), in the $4000-400 \mathrm{~cm}^{-1}$ spectral region with deuterated triglycine sulfate detector. All samples were dried at $70^{\circ} \mathrm{C}$ overnight before analysis. $\mathrm{KBr}$ pellet was used as a back ground reference. Approximately 1 $\mathrm{mg}$ of the sample was milled with approximately $100 \mathrm{mg}$ of dried $\mathrm{KBr}$ and then pressed to form a pellet for measurement.

\section{Statistical Analysis}

All experiments were carried out in triplicates $(n=3)$ and repeated 3 times. Each value is an average of 3 parallel replicates. Data were presented as mean \pm standard deviation. The data were analyzed by analysis of variance (ANOVA) to identify the significantly different groups at $(\mathrm{P}<0.05)$ by oneway ANOVA test using SPSS software statistical program (SPSS for windows ver. 21.00, USA).

\section{Results and Discussion}

In this present study $A$. platensis was grown in five different media which contain variable concentration of sucrose as carbon source. OD values were determined depending on sucrose concentration changes (Figure 1). These results suggest that the A. platensis is adapting to the mixotrophic condition. At low sucrose concentrations, stationary phase was reached in later days $\left(16^{\text {th }}\right)$ of incubation. At lower sucrose concentrations of less than $2.5 \mathrm{mM}$, the specific growth rate was lower, while the rising sucrose concentrations increased the specific growth rate (Figure 2). The highest specific growth rate $\left(0.118\right.$ day $\left.^{-1}\right)$ was detected in $2.5 \mathrm{mM}$ sucrose medium $(p<0.05)$. Similarly, in a study the highest specific growth rate was detected in mixotrophic cultivation with $2.5 \mathrm{~g} / \mathrm{L}$ glucose (Chojnacka, \& Noworyta, 2004).

In other production media the specific growth rates were detected as $0.091 \mathrm{day}^{-1}$ (with $0 \mathrm{mM}$ sucrose), 0.102 day $^{-1}$ (with $0.25 \mathrm{mM}$ sucrose), and 0.046 day $^{-1}$ (with $10 \mathrm{mM}$ sucrose), (as the specific growth rate with $50 \mathrm{mM}$ sucrose medium was lower, it was not shown in graph). In medium with high sucrose concentration (10 or $50 \mathrm{mM}$ ), the cell has mass growth and may not have gone into cell division. 2.5 fold decrease was detected in specific growth rate with 4 fold increasing sucrose concentration $(\mathrm{p}<0.05)$. In mixotrophic culture, autotrophic and heterotrophic metabolism were work together. The cyanobacteria cells were reached stationary phase rapidly and there were no significant changes in OD values. For this reason, specific growth rate of the medium with high sucrose concentration was detected lower than control condition (phototrophic cultivation).

In this present work, a higher growth rate was achieved in the mixotrophic medium than in the control condition because of low light intensity (1500 lux) and the presence of external carbon source. Even in phototrophic cultivation of A. platensis the optimal light intensity is 2500 lux, the light intensity in control condition (1500 lux or $20.25 \mu \mathrm{mol}$ photon $\mathrm{m}^{-2} \mathrm{~s}^{-2}$ ) was deficient. The light intensity in mixotrophic culture was sufficient as there was external carbon source. At high sucrose concentrations, substrate inhibition was also determined. The growth rate in the mixotrophic medium containing $10 \mathrm{mM}$ sucrose was about 2 times lower than in the control condition $(\mathrm{p}<0.05)$. As a result of reaching rapidly to the specific growth rate, substrate inhibition was detected.

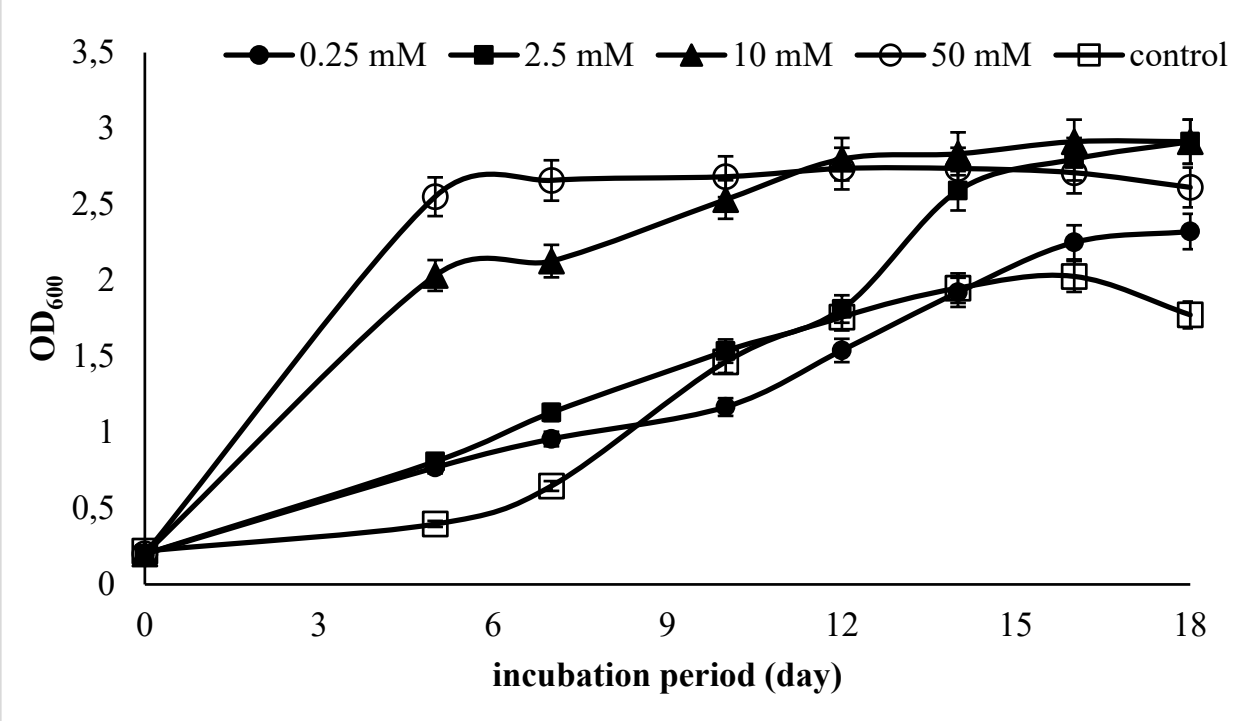

Figure 1. Variations of OD in A. platensis in different sucrose concentration medium depending on incubation period. 


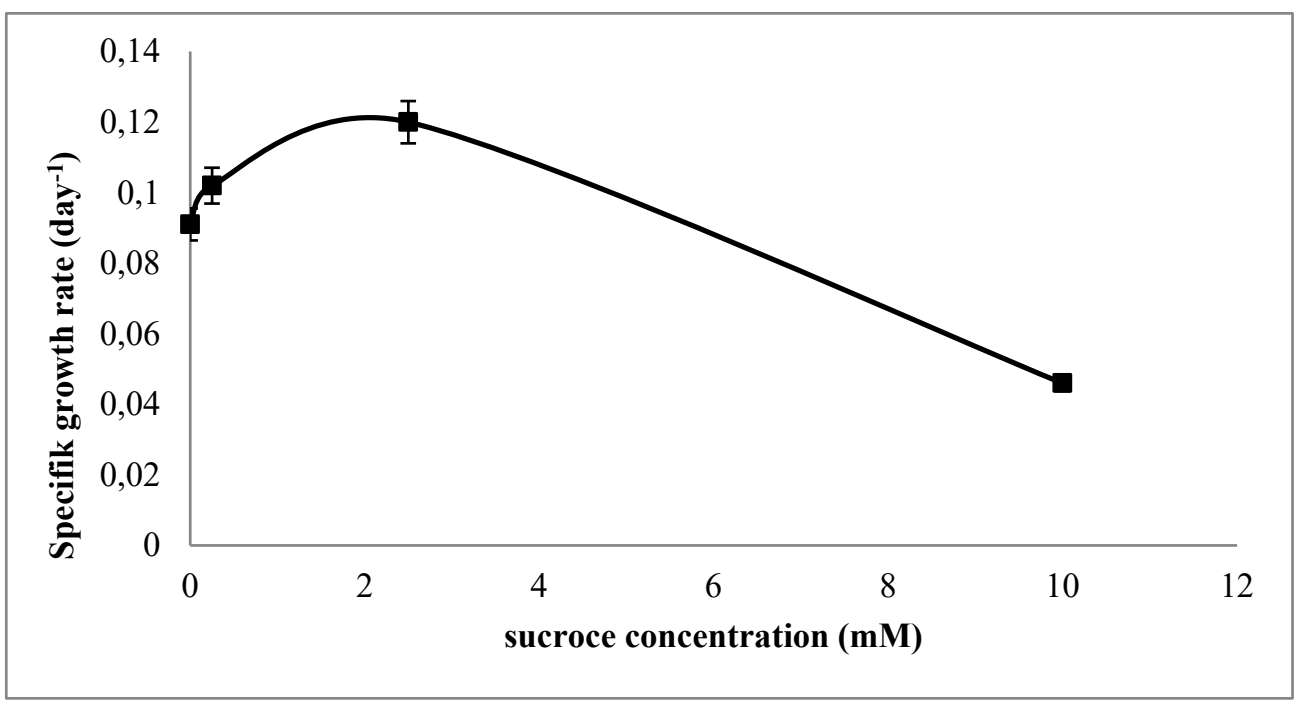

Figure 2. Specific growth rate values in varying sucrose concentrations media.

Additionally, the highest biomass productivity was detected as $1.33 \mathrm{~g} / \mathrm{L} /$ day in the medium containing $2.5 \mathrm{mM}$ sucrose. The biomass productivity of Arthrospira sp. varies from 0.06 to $4.3 \mathrm{~g} / \mathrm{L} /$ day depending on the species (Mata et al., 2010). This result shows that $A$. platensis adapted to sucrose containing medium $(2.5 \mathrm{mM})$ and reached to a high production rate. An increase was detected in biomass productivity with increasing sucrose concentration up to $2.5 \mathrm{mM}$. These results indicate that $A$. platensis cannot be adapted to high sucrose concentrations. However, the sucrose concentration up to 2.5 $\mathrm{mM}$ provided better growth than the control condition. When the medium supplemented with external carbon source, C availability can exceed cell necessities for growth and the rest carbon is directed towards lipid or carbohydrate synthesis (Lari et al., 2016). Generally specific growth rate of mixotrophic culture is the sum of phototrophic and heterotrophic metabolism because the external organic carbon promotes faster growth (Perez-Garcia et al., 2011). It can be said that, under controlled condition the specific growth rate is lower in the mixotrophic medium containing sucrose up to $2.5 \mathrm{mM}$ than the controlled media $(\mathrm{p}<0.05)$.

Different kinds of simple sugars like glucose, fructose, galactose, mannose, lactose and sucrose support the mixotrophic and heterotrophic growth of cyanobacteria with species-specific differences in uptake and assimilation mechanisms (Neilson \& Lewin, 1974; Shi et al., 1999; Sun et al., 2008). The study of the effect of different sugars and concentrations on the growth of Arthrospira sp. have shown that sucrose does not support growth in the dark but is effective in growing for certain species in the light conditions (Mühling et al., 2005). In this study, bleaching was detected in A. platensis cultures during adaptation to sucrose medium, and present study shows similarity in high sucrose concentrations (50
$\mathrm{mM}$ ). Sucrose, trehalose and glucosyglycerol are osmoprotective compounds. The cyanobacterium Synechocystis sp. has active transport mechanism for glucosyglycerol and in salt-adapted cells is mainly achieved by de novo synthesis of the transport system (Mikkat et al., 1996; Mikkat et al., 1997). The studies support that trehalose and sucrose are taken up by the cells and possesses nearly the same as glucosylglycerol.

The inhibitory effect of the $50 \mathrm{mM}$ sucrose concentration is also evident from the level of Chl-a and Chl-b content (Figure $3-4)$. The highest Chl-a content (301.173 $\pm 14.8 \mathrm{mg} / \mathrm{g}$ cell) was detected in the $2.5 \mathrm{mM}$ sucrose containing medium, while the highest Chl-b content $(42.62 \pm 1.9 \mu \mathrm{g} / \mathrm{mg}$ cell) was detected under phototrophic cultivation. The chlorophyll amount did not differ significantly between control condition and mixotrophic cultivation (with $2.5 \mathrm{mM}$ sucrose). In the study of Gim et al. (2016), the chlorophyll concentrations had no meaningful changes in mixotrophic (with $20 \mathrm{mM}$ glucose) and phototrophic cultures.

In phototrophic culture the sole carbon source was $\mathrm{CO}_{2}$ and the cyanobacteria needed chlorophyll to produce nutrient by using light and $\mathrm{CO}_{2}$. On the contrary, in mixotrophic condition $\mathrm{CO}_{2}$ was not the unique factor that supported biomass production. The mixotrophic condition is identified as "twostage" mode (Zhan et al., 2017). The first stage is heterotrophy due to high content of initial organic carbon. When the organic carbon reduces to a certain level, phototrophic metabolism gets involved as first stage. Increasing amount of Chl-a in the late days of incubation under mixotrophic condition is associated with an increase in the amount of cells and phototrophic metabolism. And also the decreasing of available organic carbon load in the medium turns the mixotrophic metabolism to phototrophic metabolism. 


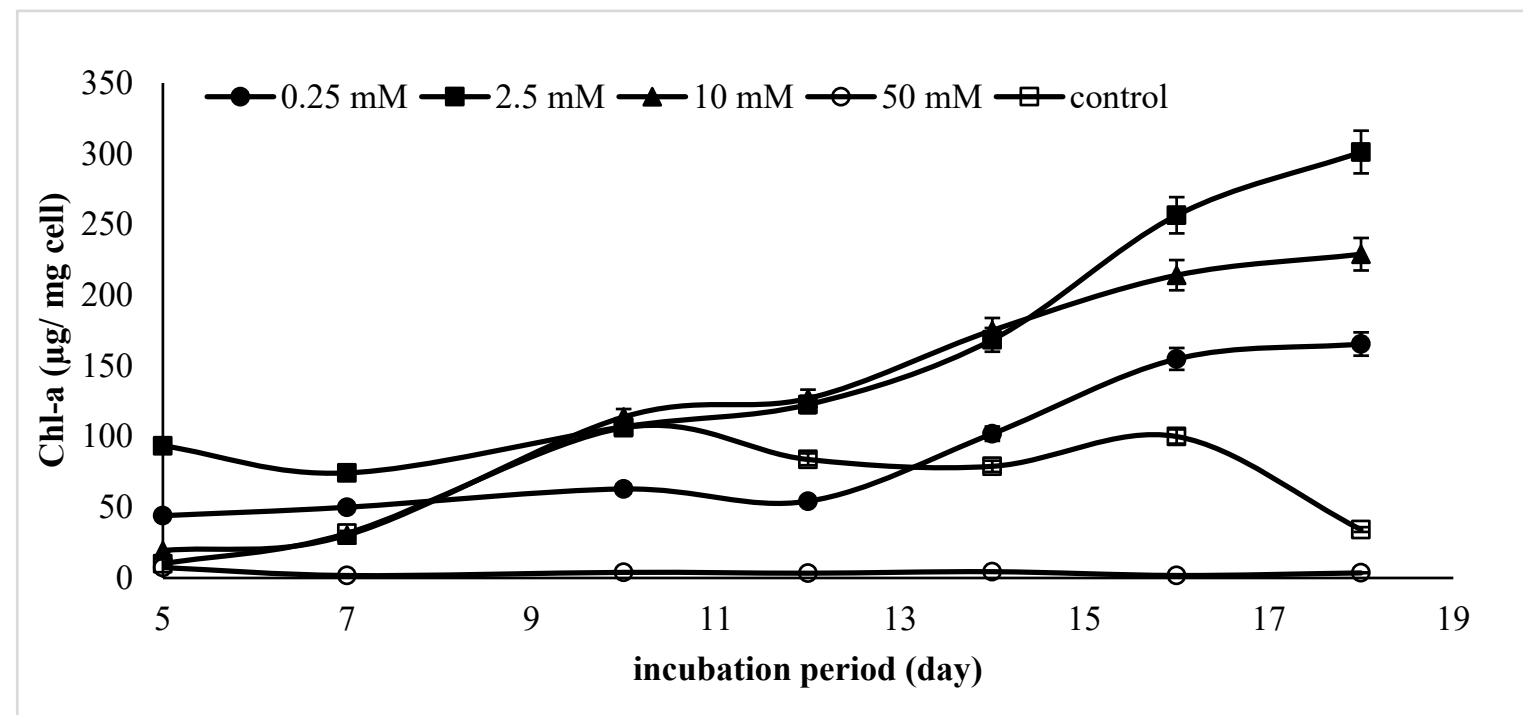

Figure 3. Chlorophyll-a content of $A$. platensis in different sucrose concentration medium depending on incubation period.

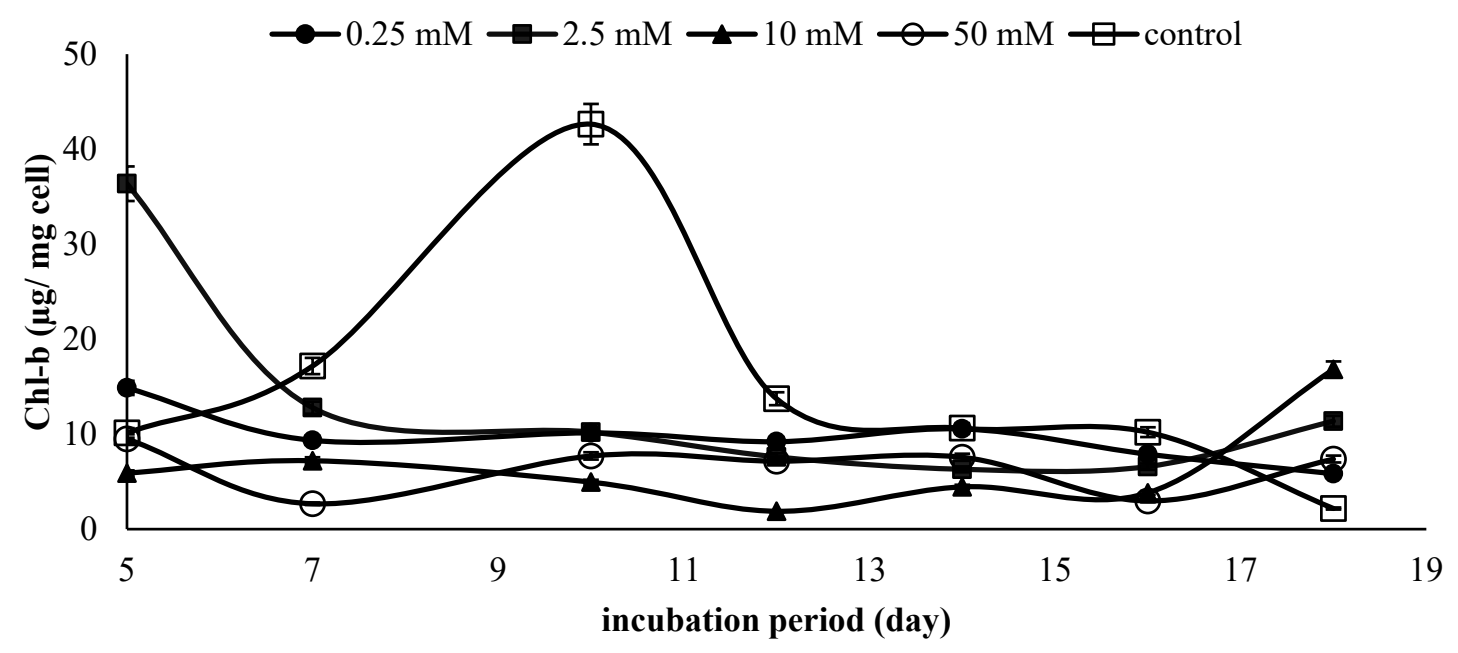

Figure 4. Chlorophyll-b content of $A$. platensis in different sucrose concentration medium depending on incubation period

There was approximately 1.5 unit overall increase in $\mathrm{pH}$ values during the incubation period (Figure 5). The determined $\mathrm{pH}$ value increase may be due to organic bases released into the medium during the production process. Only in the presence of $50 \mathrm{mM}$ sucrose, there was a decrease with a fluctuation in the $\mathrm{pH}$ value. In this medium, excess carbon source may inhibit the cellular metabolism, hence the disaccharide is not rapidly breakdown.
Insignificant lipid production was detected at minimum (control and $0.25 \mathrm{mM})$ and maximum $(50 \mathrm{mM})$ sucrose concentrations $(\mathrm{p}>0.05)$ (Figure 6). While lipid production varied with increasing sucrose concentration, the highest lipid content $(3.68 \pm 0.17 \mathrm{mg} / \mathrm{g}$ cell $)$ was determined on the $16^{\text {th }}$ day of incubation in medium containing $2.5 \mathrm{mM}$ sucrose $(\mathrm{p}<0.05)$. In control condition and the medium containing $0.25 \mathrm{mM}$ sucrose, the maximum amount of lipid was detected in the first days of incubation. In medium with high sucrose 
concentration, lipid production was increased late in the incubation days because the cells provided later adaptation. When the sucrose concentration was higher than $2.5 \mathrm{mM}$, substrate inhibition was observed. In a study two different microalgae were grown in mixotrophic culture including glucose (Cheirsilp \& Torpee, 2012). The lipid content of both strains decreased sharply when the initial glucose concentra- tion increased from 0 to $4 \mathrm{~g} / \mathrm{L}$. At above $4 \mathrm{~g} / \mathrm{L}$ of initial glucose concentration, the lipid content did not change significantly. Similarly, in the study of Lin and Wu (2015), lipid production of Chlorella sp. increased when the initial sucrose concentration increased to $0.5 \mathrm{~g} / \mathrm{L}$. When the initial sucrose concentration was higher than $0.5 \mathrm{~g} / \mathrm{L}$, the lipid production decreased.

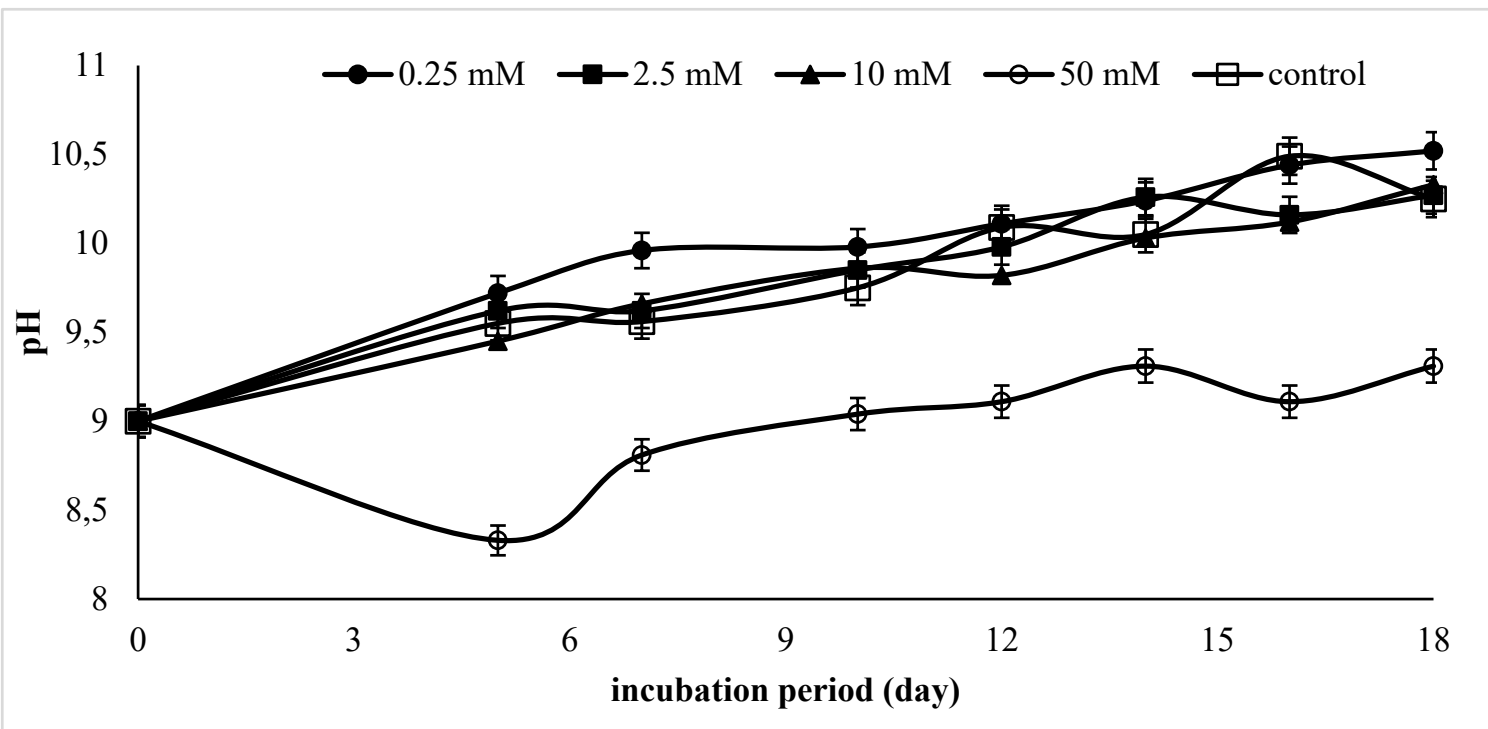

Figure 5. Variations of $\mathrm{pH}$ of $A$. platensis in different sucrose concentration medium depending on incubation period.

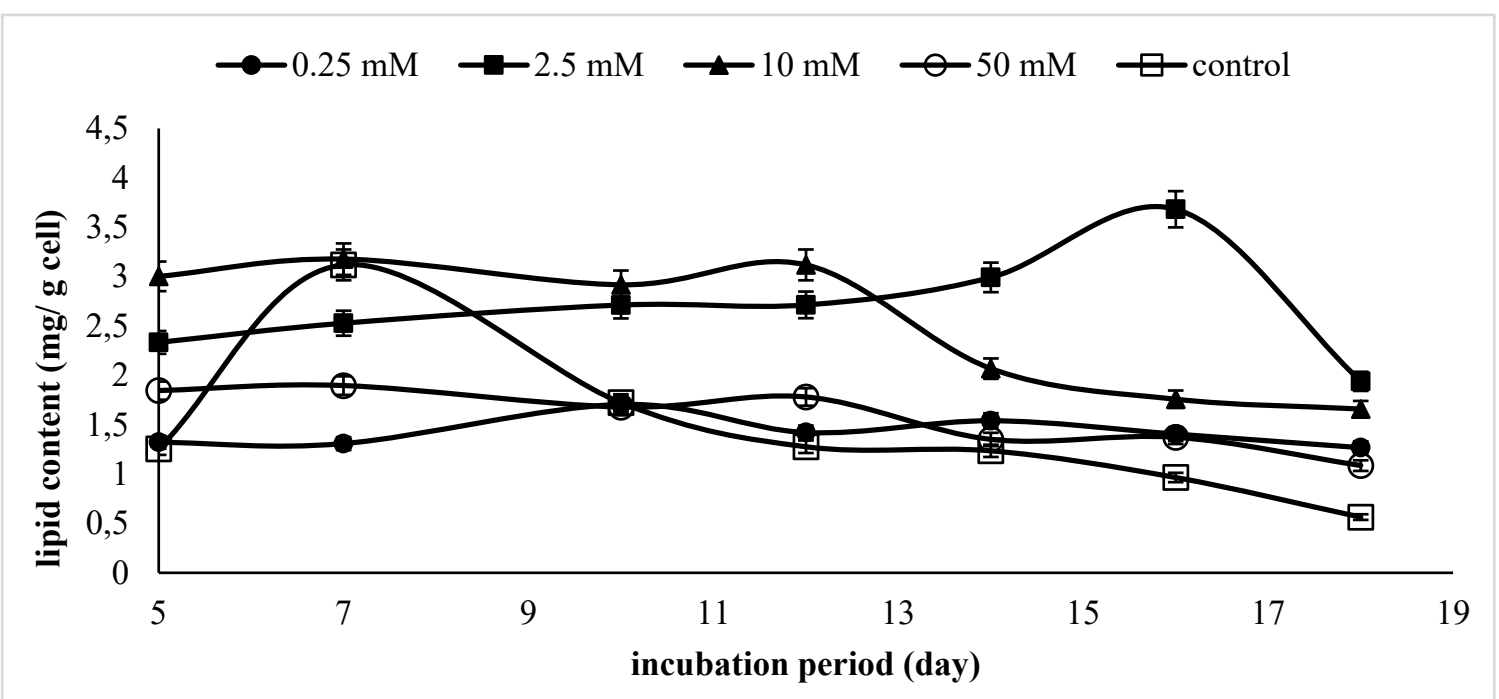

Figure 6. Lipid content of $A$. platensis in different sucrose concentration medium depending on incubation period. 
Table 1. Comparison of control condition (phototrophic) and mixotrophic culture (containing $2.5 \mathrm{mM}$ sucrose) that has been determined the best results.

\begin{tabular}{|l|l|l|}
\hline Parameters & mixotrophic culture & control (phototrophic culture) \\
\hline Specific growth rate (day $\mathbf{- 1})$ & 0.118 & 0.091 \\
\hline Biomass productivity $(\mathbf{g} / \mathbf{L} / \mathbf{d a y})$ & 1.33 & 0.153 \\
\hline Lipid content $(\mathbf{m g} / \mathbf{~ m g ~ c e l l )}$ & $3.68 \pm 0.17$ & $3.118 \pm 0.14$ \\
\hline Chl-a content $(\boldsymbol{\mu g} / \mathbf{~ m g}$ cell) & $301.173 \pm 14.8$ & $106.303 \pm 4.9$ \\
\hline Chl-b content $(\boldsymbol{\mu g} / \mathbf{~ m g ~ c e l l ) ~}$ & $36.362 \pm 1.7$ & $42.62 \pm 1.9$ \\
\hline
\end{tabular}

Values are mean \pm S.D., $\mathrm{N}=3 ;(\mathrm{p}<0.05)$.

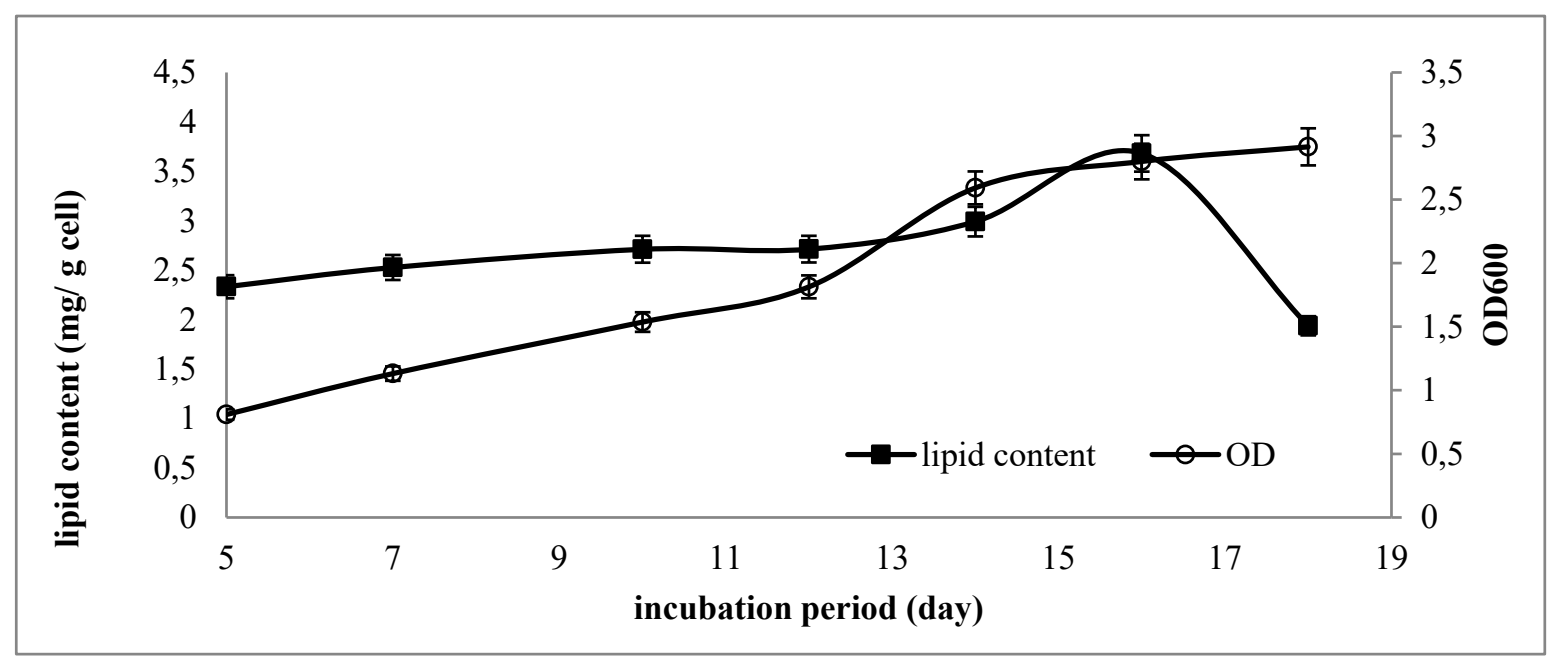

Figure 7. Lipid content and OD of A. platensis in $2.5 \mathrm{mM}$ sucrose medium depending on incubation period.

According to the obtained results, the highest specific growth rate, biomass productivity, Chl-a and lipid content was detected in mixotrophic culture that contains $2.5 \mathrm{mM}$ sucrose (Table 1). These results showed that sucrose including medium supports biomass and chlorophyll production and lipid accumulation of $A$. platensis.

In the highest lipid production condition, biomass and lipid content according to the incubation period is shown in Figure 7. According to figure it was determined that the maximum amount of lipid was obtained at the stationary phase. This can be interpreted as $A$. platensis culture grown with adaptation of sucrose has increased lipid production by entering the stress due to reduced external carbon source in the medium. In this medium on the $16^{\text {th }}$ day of incubation, external and intracellular total carbohydrate concentration was detected as $28.29 \mathrm{ppm}$ and $130.26 \mu \mathrm{g} / \mathrm{g}$ cell, respectively.

The thermal stability of the produced cell was investigated by TGA and DSC. The thermal stability of produced cell could show difference according to production medium. The produced cyanobacterium cells in different media have almost same degradation profile. In the first step, $2.19 \%$ of weight loss for phototrophic production and $4.13 \%$ of weight loss for mixotrophic production were recorded. The maximum degradation was determined in the second step for two of them. The weight loss was $52.94 \%$ and $54.74 \%$ for phototrophic and mixotrophic production, respectively. The most important difference was the cyanobacterium cell that produced in phototrophic culture has showed more rapid weight loss than produced in mixotrophic culture. That means the cyanobacterium cell produced in mixotrophic culture has higher thermal stability.

Also the functional groups of the produced cyanobacterium were investigated by FTIR. In FT-IR spectra of cyanobacterium cell produced phototrophic and mixotrophic cultures there were same peaks such as $2990-2924 \mathrm{~cm}^{-1}$ showed $\mathrm{CH}_{3}$ asymmetric stretching which was associated with lipid, carbohydrate or protein structure, $1650 \mathrm{~cm}^{-1}$ relevant with $\mathrm{C}=\mathrm{O}$ stretching on protein structure and the peak belongs to N-H bending and C-N stretching next to $1542 \mathrm{~cm}^{-1}, 1240 \mathrm{~cm}^{-1}$ related to asymmetric stretching of hydrocarbon chain and 
phospholipid structures. The peak at $2856 \mathrm{~cm}^{-1}$ was formed as a result of $\mathrm{CH}_{2}$ symmetric resonance in lipid and carbohydrate structure which was detected only cyanobacterium cell produced in mixotrophic culture FTIR spectrum. This result supports that more lipid production was produced in the mixotrophic culture.

\section{Conclusions}

In conclusion the present study suggests a new carbon source for mixotrophic culture of $A$. platensis using sucrose. A. platensis has adapted to the medium that contains low sucrose concentrations. Significant decrease was detected in specific growth rate with increasing sucrose concentration $(\mathrm{p}<0.05)$. In the mixotrophic cultivation of $A$. platensis two different metabolic pathways were active. Due to there was sufficient external carbon source in the first days of incubation, heterotrophic metabolism was used more actively. When the organic carbon source sucrose reached a critical concentration chlorophyll content started to increase. That means heterotrophic and phototrophic metabolism worked correlated. The produced cells in mixotrophic culture (with $2.5 \mathrm{mM}$ sucrose) has higher thermal stability depending on TGA. Additionally, this study showed that sucrose containing medium supports lipid production. And this result is supported by the FTIR spectrum. In this present study, it can be said that A. platensis can use sucrose as a carbon source. This result is an indication that various wastes containing sucrose such as molasses, sugar cane bagasse can also be used as a carbon source in the production medium. Thus, valuable products such as biomass, protein, and lipid can be produced more economically and hence used economically for in various industrial areas such as food, fisheries, and pharmaceuticals. The produced $A$. platensis biomass would be evaluated as protein and lipid source in aquaculture diets.

\section{Compliance with Ethical Standard}

Conflict of interests: The authors declare that for this article they have no actual, potential or perceived conflict of interests.

Ethics committee approval: There is no need ethics committee approval.

Financial disclosure: The authors declare that for this article there are no financial support.

Acknowledgments: We would like to thank Assoc. Dr. Leyla Uslu for her supply of cyanobacteria.

\section{References}

Abreu, A.P., Fernandes, B., Vicente, A.A., Teixeira, J., Dragone, G. (2012). Mixotrophic cultivation of Chlorella vulgaris using industrial dairy waste as organic carbon source. Bioresources Technology, 11, 61-66. https://doi.org/10.1016/j.biortech.2012.05.055

Bhatnagar, A., Chinnasamy, S., Singh, M., Das, K. C. (2011). Renewable biomass production by mixotrophic algae in the presence of various carbon sources and wastewaters. Applied Energy, 88(10), 3425-3431.

https://doi.org/10.1016/j.apenergy.2010.12.064

Ceron Garcia, M.C., Camacho, F.G., Mirón, A.S., Sevilla, J. F., Chisti, Y., Grima, E. M. (2006). Mixotrophic production of marine microalga Phaeodactylum tricornutum on various carbon sources. Journal of Microbiology Biotechnology, $16(5), 689$.

Cheirsilp, B., Torpee, S. (2012). Enhanced growth and lipid production of microalgae under mixotrophic culture condition: effect of light intensity, glucose concentration and fedbatch cultivation. Bioresource Technology, 110, 510-516. https://doi.org/10.1016/j.biortech.2012.01.125

Chojnacka, K., Marquez-Rocha, F.J. (2004). Kinetic and stoichiometric relationships of the energy and carbon metabolism in the culture of cyanobacterium. Biotechnology, 3(1), 21-34.

https://doi.org/10.3923/biotech.2004.21.34

Chojnacka, K., Noworyta, A. (2004). Evaluation of Spirulina sp. growth in photoautotrophic, heterotrophic and mixotrophic cultures. Enzyme and Microbial Technology, 34, 461-465.

https://doi.org/10.1016/j.enzmictec.2003.12.002

Dubois, M., Gilles, K.A., Hamilton, J.K., Rebers, P.A., Smith, F. (1956). Colorimetric method for determination of sugars and related substances. Analytical Chemistry, 28(3), 350-356.

https://doi.org/10.1021/ac60111a017

Gim, G.H., Ryu, J., Kim, M.J., Kim, P.I., Kim, S.W. (2016). Effects of carbon source and light intensity on the growth and total lipid production of three cyanobacterium 
under different culture conditions. Journal of Industrial Microbiology and Biotechnology, 43(5), 605-616.

https://doi.org/10.1007/s10295-016-1741-y

Joannesa, C., Mansaa, R.F., Yasirb, S.M., Dayouc, J. (2016). Comparative studies of cell growth of freshwater microalga Chlorella sp. in photoautotrophic, heterotrophic and mixotrophic cultures. Jurnal Teknologi (Science \& Engineering), 78(7), 83-89.

https://doi.org/10.11113/jt.v78.4349

Katiyar, R., Gurjar, B.R., Biswas, S., Pruthi, V., Kumar, N., Kumar, P. (2017). Cyanobacterium: An emerging source of energy based bio-products and a solution for environmental issues. Renewable and Sustainable Energy Reviews, 72, 1083-1093.

https://doi.org/10.1016/j.rser.2016.10.028

Kong, W.B., Yang, H., Cao, Y.T., Song, H., Hua, S.F., Xia, C.G. (2013). Effect of glycerol and glucose on the enhancement of biomass, lipid and soluble carbohydrate production by Chlorella vulgaris in mixotrophic culture. Food Technology and Biotechnology, 51(1), 62.

Lari, Z., Moradi-kheibari, N., Ahmadzadeh, H., Abrishamchi, P., Moheimani, N.R., Murry, M.A. (2016). Bioprocess engineering of cyanobacterium to optimize lipid production through nutrient management. Journal of Applied Phycology, 28(6), 3235-3250.

https://doi.org/10.1007/s10811-016-0884-6

Lichtenthaler, H.K., Wellburn, A.R. (1983). Determinations of total carotenoids and chlorophylls a and b of leaf extracts in different solvents. Biochemical Society Transactions, 11, 591-592.

https://doi.org/10.1042/bst0110591

Lin, T.S., Wu, J.Y. (2015). Effect of carbon sources on growth and lipid accumulation of newly isolated microalgae cultured under mixotrophic condition. Bioresource Technology, 184, 100-107.

https://doi.org/10.1016/j.biortech.2014.11.005

Margarites, A.C., Volpato, N., Araújo, E., Cardoso, L.G., Bertolin, T.E., Colla, L.M., Costa, J.A.V. (2017). Spirulina platensis is more efficient than Chlorella homosphaera in carbohydrate productivity. Environmental Technology, 38(17), 2209-2216.

https://doi.org/10.1080/09593330.2016.1254685

Marquez, F.J., Sasaki, K., Kakizono, T., Nishio, N. \& Nagai, S. (1993). Growth characteristics of Spirulina platensis in mixotrophic and heterotrophic conditions. Journal of Fermentation and Bioengineering, 76(5), 408-410.

https://doi.org/10.1016/0922-338X(93)90034-6

Mata, T.M., Martins, A.A., Caetano, N.S. (2010). Cyanobacterium for biodiesel production and other applications: a review. Renewable and Sustainable Energy Reviews, 14(1), 217-232.

https://doi.org/10.1016/j.rser.2009.07.020

Meireles dos Santos, A., Vieira, K.R., Basso Sartori, R., Meireles dos Santos, A., Queiroz, M.I., Zepka, L.Q., Jacob-Lopes, E. (2017). Heterotrophic cultivation of cyanobacteria: study of effect of exogenous sources of organic carbon, absolute amount of nutrients, and stirring speed on biomass and lipid productivity. Frontiers in Bioengineering and Biotechnology, 5(12), 1-7.

https://doi.org/10.3389/fbioe.2017.00012

Mikkat, S., Effmert, U. \& Hagemann, M. (1997). Uptake and use of the osmoprotective compounds trehalose, glucosylglycerol, and sucrose by the cyanobacterium Synechocystis sp. PCC6803. Archives of Microbiology, 167(2-3), 112-118.

https://doi.org/10.1007/s002030050423

Mikkat, S., Hagemann, M., Schoor, A. (1996). Active transport of glucosylglycerol is involved in salt adaptation of the cyanobacterium Synechocystis sp. strain PCC 6803. Microbiology, 142(7), 1725-1732.

https://doi.org/10.1099/13500872-142-7-1725

Mishra, S.K., Suh, W.I., Farooq, W., Moon, M., Shrivastav, A., Park, M.S., Yang, J.-W. (2014). Rapid quantification of microalgal lipids in aqueous medium by a simple colorimetric method. Bioresource Technology, 155, 330-333.

https://doi.org/10.1016/j.biortech.2013.12.077 
Mitra, D., van Leeuwen, J.H., Lamsal, B. (2012). Heterotrophic/mixotrophic cultivation of oleaginous Chlorella vulgaris on industrial co-products. Algal Research, 1(1), 40-48. https://doi.org/10.1016/j.algal.2012.03.002

Mühling, M., Belay, A., Whitton, B.A. (2005). Screening Arthrospira (Spirulina) strains for heterotrophy. Journal of Applied Phycology, 17(2), 129-135.

https://doi.org/10.1007/s10811-005-7214-8

Neilson, A.H., Lewin, R.A. (1974). The uptake and utilization of organic carbon by algae: an essay in comparative biochemistry. Phycologia, 13(3), 227-264.

https://doi.org/10.2216/i0031-8884-13-3-227.1

Nogueira, S.M.S., Souza Junior, J., Maia, H.D., Saboya, J.P.S., Farias, W.R.. (2018). Use of Spirulina platensis in treatment of fish farming wastewater. Revista Ciência Agronômica, 49(4), 599-606.

https://doi.org/10.5935/1806-6690.20180068

Ozturk Urek, R., Kerimoglu, Y. (2019). Evaluation of effects of $\mathrm{Mg}^{2+}$ and $\mathrm{Cu}^{2+}$ on pigment-metabolite production and photosystem II activity of Arthrospira platensis Gomont 1892. Turkish Journal of Fisheries and Aquatic Sciences, 19(10), 873-883.

https://doi.org/10.4194/1303-2712-v19 $10 \quad 07$

Patel, A., Gami, B., Patel, P., Patel, B. (2017). Cyanobacterium: Antiquity to era of integrated technology. Renewable and Sustainable Energy Reviews, 71, 535-547.

https://doi.org/10.1016/j.rser.2016.12.081

Perez-Garcia, O., Escalante, F.M., Bashan, L.E., Bashan, Y. (2011). Heterotrophic cultures of cyanobacterium: metabolism and potential products. Water Research, 45(1), 11-36. https://doi.org/10.1016/j.watres.2010.08.037

Rosas, V.T., Poersch, L.H., Romano, L.A., Tesser, M.B. (2018). Feasibility of the use of Spirulina in aquaculture diets. Reviews in Aquaculture, 1-12.

https://doi.org/10.1111/raq.12297

Shi, X.M., Liu, H.J., Zhang, X.W., Chen, F. (1999). Production of biomass and lutein by Chlorella protothecoides at various glucose concentrations in heterotrophic cultures. Process Biochemistry, 34(4), 341-347.

https://doi.org/10.1016/S0032-9592(98)00101-0

Silaban, A., Bai, R., Gutierrez-Wing, M.T., Negulescu, I.I., Rusch, K.A. (2014). Effect of organic carbon, C: N ratio and light on the growth and lipid productivity of cyanobacterium/cyanobacteria coculture. Engineering in Life Sciences, 14(1), 47-56.

https://doi.org/10.1002/elsc.201200219

Sivakumar, N., Sundararaman, M., Selvakumar, G. (2018). Evaluation of growth performance of Penaeus monodon (Fabricius) fed diet with partial replacement of fishmeal by Spirulina platensis (Sp) meal. Indian Journal of Animal Research, 52(12), 1721-1726.

https://doi.org/10.18805/ijar.B-3438

Sun, N., Wang, Y., Li, Y.T., Huang, J.-C., Chen, F. (2008). Sugar-based growth, astaxanthin accumulation and carotenogenic transcription of heterotrophic Chlorella zofingiensis (Chlorophyta). Process Biochemistry, 43(11), 1288-1292. https://doi.org/10.1016/j.procbio.2008.07.014

Van Wagenen, J., De Francisci, D., Angelidaki, I. (2015). Comparison of mixotrophic to cyclic autotrophic/heterotrophic growth strategies to optimize productivity of Chlorella sorokiniana. Journal of Applied Phycology, 27(5), 1775-1782.

https://doi.org/10.1007/s10811-014-0485-1

Vonshak, A., Abeliovich, A., Boussiba, S., Arad, S., Richmond, A. (1982). Production of Spirulina biomass: effects of environmental factors and population density. Biomass, 2(3), 175-185.

https://doi.org/10.1016/0144-4565(82)90028-2

Wang, S., Wu, Y., Wang, X. (2016). Heterotrophic cultivation of Chlorella pyrenoidosa using sucrose as the sole carbon source by co-culture with Rhodotorula glutinis. Bioresource Technology, 220, 615-620.

https://doi.org/10.1016/j.biortech.2016.09.010

Wang, H., Zhou, W., Shao, H., Liu, T. (2017). A comparative analysis of biomass and lipid content in five Tribonema 
sp. strains at autotrophic, heterotrophic and mixotrophic cultivation. Algal Research, 24, 284-289.

https://doi.org/10.1016/j.algal.2017.04.020

Zarrouk, C. (1966). Contribution à l'étude d'une cyanophycée. Influence de divers facteurs physiques et chimiques sur la croissance et la photosynthèse de Spirulina maxima. $(\mathrm{PhD}$ Thesis). Université de Paris, Paris, France.
Zhan, J., Rong, J., Wang, Q. (2017). Mixotrophic cultivation, a preferable cyanobacterium cultivation mode for biomass/bioenergy production, and bioremediation, advances and prospect. International Journal of Hydrogen Energy, 42(12), 8505-8517.

https://doi.org/10.1016/j.ijhydene.2016.12.021 